\title{
Auditory frequency discrimination in adult developmental dyslexics
}

\author{
S. J. FRANCE, B. S. ROSNER, P. C. HANSEN, C. CALVIN, \\ J. B. TALCOTT, A. J. RICHARDSON, and J. F. STEIN \\ University of Oxford, Oxford, England
}

\begin{abstract}
Developmental dyslexics reportedly discriminate auditory frequency poorly. A recent study found no such deficit. Unlike its predecessors, however, it employed multiple exposures per trial to the standard stimulus. To investigate whether this affects frequency discrimination in dyslexics, a traditional twointerval same-different paradigm (2I_1A_X) and a variant with six A-stimuli per trial (2I_6A_X) were used here. Frequency varied around $500 \mathrm{~Hz}$; interstimulus interval (ISI) ranged between 0 and $1,000 \mathrm{msec}$. Under 2I_1A_X, dyslexics always had larger just noticeable differences (JNDs) than did controls. Dyslexic and control JNDs were equal at shorter ISIs under 2I_6A_X, but dyslexics became worse than controls at longer ISIs. Signal detection analysis suggests that both sensory variance and trace variance are larger in dyslexics than in controls.
\end{abstract}

Developmental dyslexia, a reading disability, presents as a linguistic deficiency. Given at least nonverbal intelligence, reading achievement falls substantially below the level expected from age and intelligence(Roush, 1995; Rutter \& Yule, 1975). The disorder apparently affects upwards of $5 \%$ of the population (Shaywitz, Shaywitz, Fletcher, \& Escobar, 1990). The exact definition of the syndrome, however, remains subject to debate (see Turner, 1997).

According to a widely disseminated view, developmental dyslexia is purely linguistic in origin (Vellutino, 1987; Vellutino, Steger, \& Kandel, 1972). The disability includes poor phonological awareness, the ability to understand and manipulate letter-sound units. Dyslexic children make more errors than do normal subjects in recalling a string of rhyming letters (Shankweiler, Liberman, Mark, Fowler, \& Fischer, 1979) and also have trouble detecting rhyme in a sequence of spoken words, even before entering school (Bradley \& Bryant, 1983). Phonologicaldeficits also persist in adult dyslexics (Pennington, Van Orden, Smith, Green, \& Haith, 1990).

A complementary view (see, e.g., Stein \& Walsh, 1997) proposes that relatively low-level disturbances in visual and auditory perception in dyslexics somehow interfere with the acquisition of reading skills. Reduced phonological awareness is hypothesized to result from underspecified representations of phonemes owing to impaired acoustic

This research was supported by the Rodin Remediation Trust and by the Wellcome Research Trust. We thank Anna Corrie for her assistance during the execution of these experiments, Jonathan Winter for technical support, and Nancy Waugh for numerous helpful comments on earlier drafts of this paper. Mario Cortina Borge introduced us to nonparametric tests for differences between samples in variability. Correspondence concerning this article should be addressed to B. S. Rosner, Phonetics Laboratory, 41 Wellington Square, Oxford OX1 2JF, England (e-mail: burton.rosner@phon.ox.ac.uk). processing (Tallal, 1980). In turn, inability to read an alphabetic script further reduces phonological competence, making matters worse for dyslexics. Chinese adults who read only traditional Chinese characters are poor at phoneme deletion. Other subjects who could also read pinyin, an alphabetic form of Chinese, did perfectly well on the phonological test. This contrast is even more striking, given that spoken Chinese has numerous rhymes and segmental contrasts (Read, Zhang, Nie, \& Ding, 1986) and that the majority of Chinese ideographs actually contain phonological units (Mair, 1996). Morais (1985) found that illiterates were impaired on phoneme deletion, whereas literates within the same family were not. Illiteracy even affects patterns of cerebral cortical activation during pseudoword repetition (Castro-Caldas, Petersson, Reis, Stone-Elander, \& Ingvar, 1998).

Psychophysical experiments have revealed basic visual and auditory perceptual deficits in adult developmental dyslexics (hereafter, simply dyslexics). The impairments have been attributed mainly to poor temporal processing of stimuli (Farmer \& Klein, 1995). In vision, Lovegrove and his colleagues reported reduced transient functioning in dyslexics (see Lovegrove, 1993, for a summary). Additional psychophysical deficits have been found in motion perception (Cornelissen, Richardson, Mason, Fowler, \& Stein, 1995; Talcott, Hansen, Elikim, \& Stein, 2000; Talcott et al., 1998) and in flicker fusion (Talcott et al., 1998). The visual impairments may, in part, reflect abnormalities that have been observed in large cells (magnocells) in the visual system in many dyslexics (Galaburda \& Livingstone, 1993; Stein, 1993; Stein \& Walsh, 1997; cf. Skottun, 2000).

The increased visual thresholds in adult dyslexics are only about 2.5 times those of controls. Such relatively small effects in children, however, could compound the difficulty of acquiring sufficient reading skills and might even be signs of more serious visual disturbances that obstruct the 
acquisition of reading. In support of this argument, monocular eye patching, a distinctly nonlinguistic maneuver, produces striking gains in reading skills in dyslexic children who show visual disturbances (Stein, Richardson, \& Fowler, 2000).

In the auditory domain, dyslexics show perceptual impairments of about the same size as those in vision. Starting from observations on children with specific language impairment (Tallal \& Piercy, 1973, 1974), Tallal (1980) found that reading-disabled children performed worse than controls in judging the order of two rapidly successive 75-msec complex auditory stimuli that could differ in fundamental frequency. The poor readers were also impaired on a same-different discrimination task with those stimuli. Nagarajan et al. (1999) reported that adult dyslexics were less accurate than controls in determining the order of two rapidly successive 20-msec sinusoids that could have different frequencies. Dougherty, Cynader, Bjornson, Edgell, and Giaschi (1998) found that 10- to 12year-old dyslexics failed more often than controls to perceive dichotic pitch; no group differences appeared for pure tone detection. Other auditory deficits observed in dyslexics include reduced FM detection at lower modulation rates (Witton et al., 1998), poor FM discrimination (Stein \& McAnally, 1995) at low carrier frequencies, and deficits in detection of amplitude modulation (Hari, Sääskilahti, Helenius, \& Uutela, 1999; Menell, McAnally, $\&$ Stein, 1999). Finally, dyslexics produce abnormal neurophysiological responses to various nonspeech auditory stimuli (Baldeweg, Richardson, Watkins, Foale, \& Gruzilier, 1999; McAnally \& Stein, 1996; Nagarajan et al., 1999). ${ }^{1}$

The best-attested auditory psychophysical deficit in dyslexics is impaired auditory frequency discrimination. DeWeirdt (1988) originally observed that children who read poorly also showed reduced auditory frequency discrimination. McAnally and Stein (1996) and Hari et al. (1999) found elevated just noticeable differences (JNDs) for auditory frequency in adult dyslexics. Ahissar, Protopapas, Reid, and Merzenich (2000) made similar observations on adults with a history of reading difficulties in childhood. Using an oddball stimulus paradigm, Baldeweg et al. (1999) found that dyslexics showed poorer psychophysical detection of the deviant frequencies than did controls. The two groups, however, showed no differences in detecting oddball variation in the duration of $1-\mathrm{kHz}$ tones.

Hill, Bailey, Griffiths, and Snowling (1999), however, recently reported no deficit in frequency discrimination in dyslexics. They made no attempt to explain the discrepancy between their findings and all prior results. The present paper examines one possible source of the contradiction between the results of Hill et al. and those of numerous other investigators.

All previous experiments on frequency discrimination in poor readers have used two-interval same-different (2IAX) paradigms, except for the work of Hill et al. (1999). Instead, they utilized a four-interval forced-choice (4IFC) procedure. In the first and fourth intervals of each trial, their subjects heard two stimuli known to be identical. The subject indicated whether the second or third interval contained a stimulus that differed from the initial and final sounds. In a 2IAX test, however, subjects do not get repeated exposures to known identical stimuli on each trial. Many dyslexics have relatively short auditory digit spans, a fact known at least since Torgeson's (1978) review of studies on serial memory in children. Defects in auditory memory could make the 2IAX procedure difficult for dyslexics. Repeated exposure to known identical stimuli on each trial, as in the 4IFC task, might counteract such deficits and improve dyslexics' frequency discrimination.

To test this hypothesis, we measured auditory frequency discrimination in dyslexics and controls under two conditions, using pure tones centered around $500 \mathrm{~Hz}$. In the first condition, we employed McAnally and Stein's (1996) roving 2IAX adaptive method, since this had revealed poor frequency discrimination by dyslexics. For clarity, we designate this paradigm as 2I_1A_X, two-interval same-different discrimination with one A-stimulus. In the other main condition, designated 2I_6A_X, six successive, identical A-stimuli occurred on each trial. The subject made a same-different judgment on the succeeding $\mathrm{X}$-stimulus, compared with the six preceding A-tones. Studies on detection have utilized multiple observations, which improve performance (see Green \& Swets, 1966, chap. 9). Subjects can apparently average the strengths of multiple sensory impressions on a given trial, thereby reducing signal variance. We know of no previous use in experiments on discrimination. We expected that both dyslexics and controls would have lower JNDs under 2I_6A_X, as compared with 2I_1A_X. The results of Hill et al. (1999) suggested that the dyslexics might even perform as well as the controls under the 2I_6A_X procedure.

All previous 2I_1A_X studies on frequency discrimination by dyslexics used relatively long interstimulus intervals (ISIs), $300 \mathrm{msec}$ or more. That could have magnified any effect of poor auditory memory in dyslexia. To examine this possibility, we varied the ISI between the two stimuli on each 2I_1A_X trial. The ISIs were 0, 10, 400, and $1,000 \mathrm{msec}$. We specify a particular ISI such as $400 \mathrm{msec}$ under this condition as 2I_1A_X-400. Similarly, we varied the ISI between the end of the six identical A-stimuli and the start of the X-stimulus on each 2I_6A_X trial. On the basis of pilot work (France, Hansen, Rosner, \& Stein, 1997), we set the ISIs for the 2I_6A_X condition at 10, 200,400 , and $1,000 \mathrm{msec}$. A particular ISI such as $10 \mathrm{msec}$ under this condition is labeled as 2I_6A_X-10. Within a condition, the ISI varied randomly from trial to trial, thereby increasing stimulus uncertainty. All the subjects experienced both forms of discrimination, with order balanced across subjects.

\section{METHOD}

\section{Stimuli}

Sinusoidal stimuli were generated with Tucker-Davis Technologies System II equipment, controlled by a PC. Frequency ranged from 480 to $580 \mathrm{~Hz}$ in 1-Hz steps. Each stimulus lasted $300 \mathrm{msec}$ 
onset to offset, had cosine-gated 20-msec rise/fall times, and was presented binaurally at $63 \mathrm{~dB}$ SPL through headphones.

\section{Procedure}

Tests were conducted in a quiet room. Each subject participated in two experimental sessions of no more than 40 min each, on separate days. One session was devoted to Condition 2I_1A_X, with ISIs of $0,10,400$, or $1,000 \mathrm{msec}$ between the $\mathrm{A}$ - and the $\mathrm{X}$-tone. The other session was devoted to Condition 2I_6A_X, with ISIs between the last A-tone and the X-tone of 10, 200, 400, or 1,000 msec. The interval between successive A-tones was $50 \mathrm{msec}$.

A trial began with a 400-msec visual prompt on the computer VDU. Immediately thereafter, one A-tone (2I_1A_X) or six identical A-tones (2I_6A_X) occurred, followed by an ISI and then one Xtone. The subject decided whether the A-tone(s) and the X-tone had the same or a different pitch. No time limit was placed on responding. All A-tones on any 2I_6A_X trial were known to be identical. The experimenter registered the subject's response, using a mouse. We followed McAnally and Stein's (1996) roving-level procedure. The standard frequency in 2I_1A_X and 2I_6A_X was randomly set on each trial at a value between 480 and $519 \mathrm{~Hz}$; the comparison frequency always exceeded that of the standard. The order of presentation of standard and comparison frequencies varied randomly across the 2I_1A_X pairs and across the six initial A-tones and the X-tone for 2I_6A_X.

A weighted adaptive paradigm based on Kaernbach's (1991) procedure was used. Within a condition, each of the four ISIs occurred separately in individual initial runs. A starting difference of $60 \mathrm{~Hz}$ was adjusted in a 6-Hz-down-3-Hz-up procedure until two failures to detect a difference had occurred. After the initial runs, the ISI values occurred in four randomly interleaved $1-\mathrm{Hz}$-down-2-Hz-up staircases. The starting frequency difference for a given ISI was set $3 \mathrm{~Hz}$ above the final value in the corresponding initial run. Each staircase continued through 10 reversals. The frequency JND $(\Delta F)$ for each ISI was the arithmetic mean of the last 8 reversals. In addition, catch trials, comprising an X-tone differing by 0 or $60 \mathrm{~Hz}$ from the A-tone(s), occurred randomly $20 \%$ of the time to check for unduly high false alarm and miss rates. Visual feedback was given on the VDU on these check trials, but not otherwise. Data were eliminated from subjects whose error rates exceeded .25 on the check trials in either the 2I_1A_X or the 2I_6A_X condition.

\section{Subjects}

Twenty adult dyslexics and 20 nondyslexic adult controls participated. All were unpaid volunteers with no reported hearing problems, confirmed by a brief audiometric screening test. Educational psychologists had positively diagnosed all dyslexics within the past 5 years, using among other criteria, the conventional one of a significant discrepancy between reading ability and IQ. The controls reported no history of reading problems. To assay reading ability, we administered to each subject the reading and spelling subscales from the Wide Range Achievement Test- Revised (WRAT-R; Jastak \&
Wilkinson, 1984). We also measured their performance on the auditory digit span, block design, and picture arrangement subtests of the revised Wechsler Adult Intelligence Scale (WAIS-R; Wechsler, 1981).

Half the dyslexics and half the controls received the experimental conditions in the order 2I_1A_X, then 2I_6A_X. The other subjects underwent the two conditions in the opposite order. Assignment of subjects to orders was unsystematic.

\section{RESULTS}

The samples of psychometric and psychophysical data were often not Gaussian. Except for three initial overall analyses of the frequency discrimination data with general linear models (GLMs), exact nonparametric tests were used for statistical comparisons. Significant correlations between many of the dependent variables made Bonferroni corrections inappropriate, since these assume independent trials. To guard against errors of Type I, we set $\alpha$ at .025 , using two-tailed tests.

One dyslexic performed 2.3 standard deviations below average on picture arrangement and 1.3 standard deviations below average on block design. These scores raised serious doubts about the subject's original diagnosis as a discrepant reader (see Siegel, 1992; Stanovich, 1991). We dropped further consideration of this subject's data. Of the remaining 19 dyslexics, the 2 who had the shortest digit spans and 1 other subject produced error rates above .25 on the 2I_1A_X check trials. The data from these 3 dyslexics were also eliminated from further consideration. This left 16 dyslexics and 20 controls.

\section{Psychometric Findings}

Table 1 shows the psychometric results for each group. To facilitate comparisons between the WRAT-R and the WAIS-R data, the former have been transformed to standardized scores with a mean of 10 and standard deviation of 3 . The WRAT-R tests confirmed that the dyslexics were poor readers. They scored significantly lower than the controls on reading (exact Mann-Whitney $U=1.500$, $p<.001)$ and spelling $(U=0.000, p<.001)$. One dyslexic performed slightly better than the lowest control on reading. The groups did not overlap on spelling, however, giving a value of zero for $U$. As was expected, the dyslexics had a shorter digit span than did the controls $(U=61.0, p=.001)$. The two nonverbal performance subtests of the WAIS-R showed no group differences.

Table 1

Psychometric Results (Means and Standard Deviations) for Controls and Dyslexics

\begin{tabular}{|c|c|c|c|c|}
\hline \multirow[b]{2}{*}{ Measure } & \multicolumn{2}{|c|}{ Controls $(n=20)$} & \multicolumn{2}{|c|}{ Dyslexics $(n=16)$} \\
\hline & $M$ & $S D$ & $M$ & $S D$ \\
\hline WRAT-R reading $\mathrm{a}$ & 13.6 & \pm 0.7 & $7.6 * *$ & \pm 3.2 \\
\hline WRAT-R spellinga & 13.5 & \pm 1.3 & $6.3 * *$ & \pm 3.1 \\
\hline WAIS-R digit span & 12.4 & \pm 2.4 & $8.7 * *$ & \pm 2.7 \\
\hline WAIS-R block design & 14.1 & \pm 3.0 & 13.7 & \pm 2.7 \\
\hline WAIS-R picture arrangement & $11.8 * *$ & \pm 3.0 & 10.8 & \pm 2.5 \\
\hline
\end{tabular}

aTransformed to a mean of 10 and a standard deviation of $3 . * * p<.01$ (two-tailed). 


\section{Analysis of JNDs With General Linear Models}

We performed three analyses of the $\Delta F$ values with GLMs. In all three cases, after transformation by $y=$ arc$\tan \left(\mathrm{JND}^{0.9}\right)$, the data satisfied Levene's test for homogeneity of variances and Mauchly's test of sphericity.

The first GLM was applied to the 2I_1A_X data. The within-subjects factor was ISI $(0,10,400$, or $1,000 \mathrm{msec})$. Status (control, dyslexic) and order (2I_1A_X first, 2I_6A_X first) were between-subjects factors. Box's test of equality of covariance matrices was met. ISI had a significant effect $[F(3,96)=3.429, p<.025]$, as did status $[F(1,32)=17.769, p<.001]$. No order effect and no significant interactions appeared. Marginal means indicated that $\Delta F$ increased for both groups as ISI lengthened and that dyslexics had higher thresholds than did controls.

For the GLM analysis of the 2I_6A_X results, the within-subjectsfactor was ISI $(10,200,400$, or $1,000 \mathrm{msec})$, and status and order were between-subjects factors. Box's test of equality of covariance matrices proved significant $[F(30,2527.455)=2.095, p<.001]$. Reliable effects appeared for ISI $[F(3,96)=13.389, p<.001]$ and status $[F(1,32)=11.753, p=.002]$. As under 2I_1A_X, $\Delta F$ seemed to increase for both groups as ISI lengthened, and the dyslexics had higher thresholds than did the controls. All interactions involving ISI fell well short of significance. The failure of Box's test impugns none of these results. Both order $[F(1,32)=3.601, p=.067]$, and status $\times$ order $[F(1,32)=3.266, p=.080]$, however, approached significance. Had Box's test been satisfactory, one or both $F$ values could have become significant. ${ }^{2}$

The final GLM used data where the ISI was the same under conditions 2I_1A_X and 2I_6A_X.Within-subjects factors were condition (2I_1A_X, 2I_6A_X) and ISI (10, 400 , or $1,000 \mathrm{msec}$ ), the values being common to both conditions. Order and status were between-subjects factors. Box's test was satisfactory. Significant main effects emerged for condition $[F(1,32)=32.194, p<.001]$, ISI $[F(2,64)=$ $19.821, p<.001]$, and status $[F(1,32)=18.249, p<.001]$. A significant interaction occurred between ISI and condition $[F(2,64)=12.426, p<.001]$. The marginal means indicated that performance grew worse at longer ISIs and that the dyslexics had larger JNDs than did the controls. The 2I_6A_X condition yielded lower JNDs than did the 2I_1A_X condition. The ISI $\times$ condition interaction arose because differences between JNDs under the two conditions were largest at the 10 -msec ISI and smallest at the 1,000 -msec ISI.

To summarize, the GLM analyses yielded four findings. First, $\Delta F$ increased as ISIs lengthened for all the subjects. Second, the dyslexics generally had larger values of $\Delta F$ than did the controls. Third, $\Delta F$ in the 2I_1A_X condition was larger than that under 2I_6A_X. Fourth, condition interacted with ISI. At shorter ISIs, $\Delta F$ values were larger under 2I_1A_X than under 2I_6A_X, whereas such differences at longer ISIs were negligible.

The discrimination data were combined across order, since it had produced no reliable main effects. Figure 1 displays box plots of the JNDs for the controls and the dyslexics at each ISI. The upper and lower panels contain the results for 2I_1A_X and 2I_6A_X, respectively. Each plot displays all outliers beyond the 10th and 90th percentiles. The data for the dyslexics are positively skewed. For ease of comparison, Table 2 presents the median $\Delta F$ values and the resulting Weber fractions for each group under each ISI for each condition.

\section{Between-Group Differences}

Figure 1 shows that the median values of $\Delta F$ for the dyslexics exceed those for the controls at all ISIs under the 2I_1A_X condition, agreeing with the main effect of status in the GLM results. For more detailed analysis, we compared $(\Delta F)$ values between the controls and the dyslexics at each ISI under each condition. Under condition 2I_1A_X, the comparisons between groups were significant at each ISI (exact Mann-Whitney $U=36.0,50.0,36.5$, and 51.0, $N=36, p<.001$, for the $0-, 10-, 400-$, and 1,000msec ISIs, respectively). For 2I_6A_X, however, group differences were significant at only the two longest ISIs $(U=66.5, N=36, p<.0025$, and $U=50.0, N=36, p<$ .001 , at the 400 - and 1,000-msec ISIs, respectively). Open diamonds in Figure 1 indicate these six significant differences. We also performed exact Wald-Wolfowitz runs tests on the differences between the dyslexics and the controls for 2I_6A_X-10 and 2I_6A_X-200. These tests confirmed the two negative Mann-Whitney results and indicated that the samples from the two groups were similarly shaped.

The dyslexics and the controls did not differ significantly in incorrect response rates on the check trials under 2I_1A_X $(U=157.0, N=36, p>.025)$. They differed marginally, however, under 2I_6A_X $(U=900.5, N=36$, $p=.026)$, with the dyslexics responding more erratically.

Figure 1 suggests that JNDs for dyslexics are more variable than those for controls at each ISI under each condition. In each set of data, some dyslexic JNDs equal those for some controls. We tested each of the eight differences in variability between groups, using the Ansari-Bradley statistic in a nonparametric procedure (see Randles \&

Table 2

Median Just Noticeable Differences $\Delta F$ and Weber Fractions $\Delta F / F$ for Controls $(n=20)$ and Dyslexics $(n=16)$

\begin{tabular}{|c|c|c|c|c|}
\hline \multirow{2}{*}{$\begin{array}{l}\text { Interstimulus } \\
\text { Interval (msec) }\end{array}$} & \multicolumn{2}{|c|}{$\Delta F(\mathrm{~Hz})$} & \multicolumn{2}{|c|}{$\Delta F / F$} \\
\hline & Controls & Dyslexics & Controls & Dyslexics \\
\hline \multicolumn{5}{|c|}{ Condition 2I_1A_X } \\
\hline 0 & 6.8 & 16.5 & 0.014 & 0.033 \\
\hline 10 & 7.1 & 19.1 & 0.014 & 0.038 \\
\hline 400 & 7.0 & 23.6 & 0.014 & 0.047 \\
\hline 1,000 & 7.5 & 15.9 & 0.015 & 0.032 \\
\hline \multicolumn{5}{|c|}{ Condition 2I_6A_X } \\
\hline 10 & 4.1 & 5.2 & 0.008 & 0.010 \\
\hline 200 & 4.3 & 6.2 & 0.009 & 0.012 \\
\hline 400 & 5.7 & 9.4 & 0.011 & 0.019 \\
\hline 1,000 & 6.5 & 15.1 & 0.013 & 0.030 \\
\hline
\end{tabular}




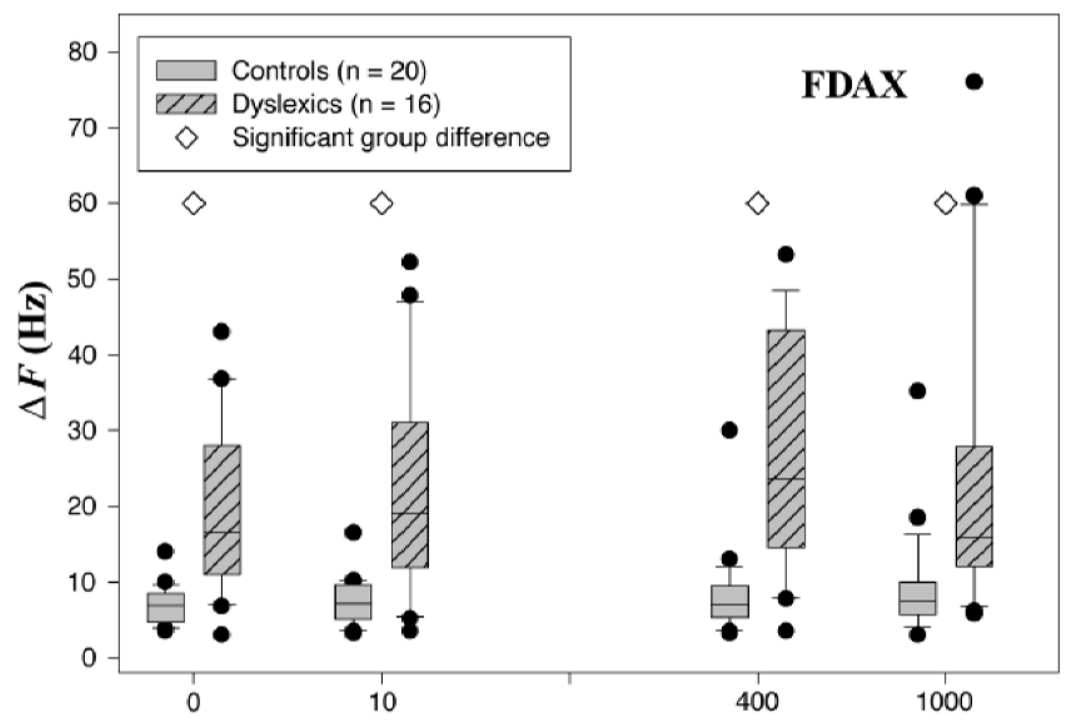

Single Comparison Tone: Delay to Target (ISI in msec)

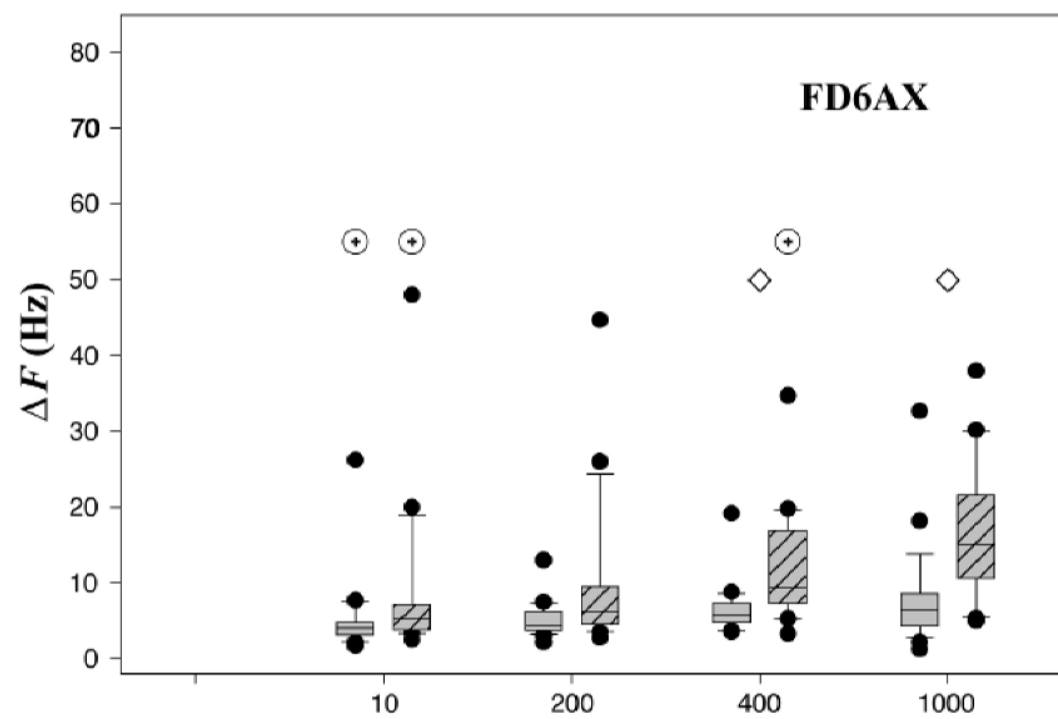

Six Comparison Tone: Delay to Target (ISI in msec)

Figure 1. Box plots of auditory roving-level just noticeable frequency differences $\Delta F$ for adult developmental dyslexics and controls at increasing interstimulus intervals (ISIs) under the 2I_1A_X (upper panel) and the 2I_6A_X (lower panel) conditions. All outlying datapoints shown are below the 10 th and above the 90 th percentile. Diamond, significant difference between groups; encircled plus sign, significant difference between conditions.

Wolfe, 1980,pp. 315-320). None of the eight tests yielded significant results.

\section{Within-Group Differences: Condition and ISI}

Exact Wilcoxon tests were used to examine the effect of condition in more detail. We compared $\Delta F$ values across the 2I_1A_X and 2I_6A_X conditions, within each group at each common ISI $(10,400$, and 1,000 msec).
Three of the six tests were significant, as is indicated by encircled crosses in the bottom panel of Figure 1. One was for the controls at the 10-msec ISI $(z=3.300, N=20, p<$ $.001)$; the other two were for the dyslexics at the ISIs of 10 $\operatorname{msec}(z=3.294, N=16, p<.001)$ and $400 \mathrm{msec}(z=3.413$, $N=16, p<.001)$. Performance improved in all three instances under 2I_6A_X, confirming the favorable effect of this paradigm found in the third GLM analysis. 
Although ISI was statistically significant in the GLM analysis of the 2I_1A_X data, that effect is not obvious in Figure 1. Within both dyslexics and controls, however, the 2I_6A_X median $\Delta F$ values seem comparable at the 10and 200-msec ISIs and then increase under 2I_6A_X-400 and 2I_6A_X-1000. To follow up these observations, we tested the differences between $\Delta F$ at the shortest and longest ISIs within each group under each condition. Under2I_1A_X-0, $\Delta F$ was significantly lower than under 2I_1A_X-1000 for the controls $(z=2.777, N=20, p=.021)$, but not for the dyslexics. Under 2I_6A_X-10, performance was better than under 2I_6A_X-1000 for both the controls $(z=3.018, N=20, p=.001)$ and the dyslexics $(z=$ $3.464, N=16, p<.001)$. These findings confirm the separate GLM analyses of the 2I_1A_X and the 2I_6A_X data. We examined the differences between pairs of successively longer ISIs under 2I_6A_X. The control $\Delta F$ values rose significantly between 2I_6A_X-200 and 2I_6A_X$400(z=2.427, N=20, p<.025)$. For the dyslexics, $\Delta F$ increased significantly between 2I_6A_X-200 and 2I_6A_X-400 $(z=2.482, N=16, p=.011)$ and again between 2I_6A_X-400 and 2I_6A_X-1000 $(z=2.689, N=$ $16, p=.005)$. In brief, increases in ISI made performance in both groups clearly worse under condition 2I_6A_X but had only a modest effect under condition 2I_1A_X on the controls alone. The dyslexics performed equally poorly at all ISIs under the 2I_1A_X condition.

\section{Correlational Analyses}

To evaluate the consistency of individual performance, we computed Spearman correlation coefficients on $\Delta F$ values within each group across all pairs of the eight combinations of conditions and ISI. We also calculated withingroup correlation coefficients between $\Delta F$ and the scores on the psychometric variables. Appendices A and B give the results for the controls and the dyslexics, respectively.

For the controls, 21 of the 28 correlations between $\Delta F$ values were positive, 19 at the .01 level and 2 at the .025 level. The dyslexics were less consistent, with only 7 significant correlations at the .01 level and 2 significant at the .025 level. For both groups, more correlations were significant at the .01 level within than between conditions.

A negative correlation between $\Delta F$ and psychometric results indicates that better psychophysical performance tends to accompany higher psychometric scores. The control subjects produced only one significant correlation between frequency discrimination and psychometric results: a negative correlation between the 2I_6A_X-1000 $\Delta F$ values and picture arrangement scores $\left(r_{\mathrm{s}}=-.51, N=20\right.$, $p<.025)$. The dyslexics, however, produced significant negative correlations between reading scores and $\Delta F$ under longer ISIs (for 2I_1A_X-400, $r_{\mathrm{s}}=-.68, N=16$, $p<.01$; for 2I_6A_X-200, $r_{\mathrm{s}}=-.60, N=16, p<.025$; for 2I_6A_X-1000, $\left.r_{\mathrm{s}}=-.59, N=16, p<.025\right)$. No significant correlations occurred between the dyslexics' $\Delta F$ values and scores for picture arrangement, block design, or spelling. In contrast to the controls, the dyslexics yielded significant negative correlations between auditory digit span and $\Delta F$ values for 2I_1A_X-10 $\left(r_{\mathrm{s}}=-.58, N=\right.$ $16, p<.025)$ and 2I_1A_X-400 $\left(r_{\mathrm{s}}=-.62, N=16, p<.01\right)$. (The latter may have affected the correlation between $\Delta F$ under2I_1A_X-400 and reading scores.) The correlations between digit span and thresholds under 2I_1A_X-1000, 2I_6A_X-10, and 2I_6A_X-200 were marginally significant $(.025<p<.05)$ for dyslexics. Finally, we combined data across the two groups and calculated the correlation between digit span and the difference between JNDs at 10 and 1,000 msec under2I_6A_X. This correlation was significant $\left(r_{\mathrm{s}}=-.41, N=36, p<.025\right) .^{3}$

Correlational analysis, then, yielded four main findings. First, the dyslexics seemed less consistent in their psychophysical performance than did the controls. Second, within each condition and each group, JNDs at one ISI tended to predict JNDs at the other ISIs; weaker relationships prevailed between JNDs across conditions. Third, JNDs and reading were related within the dyslexics. Fourth, digit span was related to JNDs, especially among the dyslexics.

\section{DISCUSSION}

We have confirmed previous reports of higher JNDs for auditory frequency in dyslexics (Ahissar et al., 2000; Baldeweg et al., 1999; Hari et al., 1999; McAnally \& Stein, 1996) and in poor readers (DeWeirdt, 1988) in a two-interval procedure with one A-stimulus per trial (2I_1A_X). The previous results were obtained at ISIs of $300 \mathrm{msec}$ or more. We extended this past work by finding larger JNDs in dyslexics than in controls at ISIs of 0 and $10 \mathrm{msec}$ in the 2I_1A_X paradigm.

Under condition 2I_6A_X, the subjects received six Astimuli per trial. As was expected, dyslexic and control JNDs both decreased. The difference thresholds for the two groups, however, were statistically indistinguishable at ISIs of 10 and $200 \mathrm{msec}$. This result suggests that the multiple observations available in the 4IFC experiment by Hill et al. (1999) helped to bring their dyslexics' JNDs down to control levels. Our dyslexics, however, were significantly less sensitive than the controls under2I_6A_X at the 400msec ISI, precisely the value that Hill et al. had employed. Two further differences between their experiment and ours may explain this fact. Hill et al. offered feedback on every trial; like other previous investigators, we employed no feedback (except on catch trials). All the dyslexics studied by Hill et al. were university undergraduates. Our dyslexic subjects had more variable educational backgrounds and, on average, may not have been so well compensated.

Hill et al. (1999) found average Weber fractions for frequency discrimination at $1 \mathrm{kHz}$ of about 0.003 and 0.004 for controls and dyslexics, respectively. These are below the values of 0.009 and 0.020 reported by McAnally and Stein (1996). Hence, the 2I_1A_X paradigm seems generally harder than 4IFC. Although Hari et al. (1999) also 
obtained a Weber fraction of 0.003 for controls, their dyslexics produced a value of 0.009 . This suggests that the 2I_1A_X procedure may be disproportionately harder for dyslexics than is 4IFC. Ahissar et al. (2000) used an ISI of $800 \mathrm{msec}$ and obtained remarkably high Weber fractions of 0.037 and 0.072 for controls and dyslexics, respectively. These values are larger than any we report in Table 2. Our 2I_1A_X Weber fractions, however, exceed the rest of those obtained previously and may reflect the additional stimulus uncertainty induced by varying the ISI within a session. Finally, in all past studies and our own, control JNDs are larger than those highly trained observers produce (see Weir, Jesteadt, \& Green, 1977).

\section{Signal Detection Analysis: Sensory Variance}

Macmillan, Goldberg, and Braida's (1988) signal detection model of perceptual resolution (hereafter, the MGB model) is applicable to our data. The MGB model follows Durlach and Braida's (1969) preliminary theory of intensity perception in distinguishing three types of psychophysical variance: sensory, trace, and context variance. Sensory variance represents unavoidable internal noise in the processing of an individual stimulus. Trace variance is due to variability in memory processes. It increases with the time between successive stimuli presented for comparison in a discrimination trial. Discrimination deteriorates as ISI increases. Context variance, another memory component, arises only when the set of experimental stimuli is sufficiently small that subjects can effectively label individual items. Context variance increases with the range of the stimuli and makes rovinglevel discrimination between members of the set worse than fixed-level discrimination. Our 2I_1A_X and the 2I_6A_X procedures employed sets of stimuli large enough to prevent effective labeling. According to the MGB model, our subjects would have used the trace mode of discrimination, where only sensory and trace variance affect sensitivity. Therefore, we will not consider context variance any further.

Sensory variance $\beta^{2}$ and trace variance $A T$ combine independently and additively in the MGB model to yield total variance, symbolized here as $v$ :

$$
v=\beta^{2}+(A T),
$$

where $T$ is the ISI and $A$ is a constant. If $a$ is the mean sensation difference between two stimuli, differential sensitivity $\left(d^{\prime}\right)$ varies inversely with the square root of $v: d^{\prime}=a /(\sqrt{ } v)$. Increases in either sensory or trace variance would raise $v$ and therefore reduce $d^{\prime}$. Reduced sensitivity, in turn, would increase the JND for frequency, $\Delta F$.

Abnormalities in low-level auditory neural responses to single stimuli would be manifested as increased sensory variance in the MGB model. If dyslexia is associated with such abnormalities, frequency discrimination in dyslexics should still be impaired at ISIs well below those used previously by McAnally and Stein (1996), Hari et al. (1999), Baldeweg et al. (1999), and Ahissar et al. (2000). At short
ISIs, trace variance becomes minimal and can be ignored. Table 2 shows that the dyslexics had larger JNDs than did the controls under 2I_1A_X-0 and 2I_1A_X-10. This eliminates trace variance as the sole source of impaired frequency discrimination in dyslexics.

In a roving-level 2I_1A_X paradigm, the subject must attend to both stimuli on each trial. Assume now that sensory variance contains independent, additive components for the $\mathrm{A}$ - and the $\mathrm{X}$-stimuli. Total sensory variance is then

$$
\beta_{1}^{2}=\beta_{\mathrm{A}}^{2}+\beta_{\mathrm{X}}^{2} .
$$

Suppose that the subject efficiently averages the strengths of sensory impressions from the successive A-tokens on each 2I_6A_X trial, similar to the averaging that seems to occur in detection tasks. For the 2I_6A_X paradigm, total sensory variance becomes

$$
\beta_{6}^{2}=\frac{\beta_{\mathrm{A}}^{2}}{6}+\beta_{\mathrm{X}}^{2} .
$$

in the MGB model. If the components of sensory variance in the 2I_1A_X paradigm are equal, so that $\beta_{\mathrm{A}}^{2}=\beta_{\mathrm{X}}^{2}$, Equations 2 and 3 yield

$$
\frac{\beta_{1}^{2}}{\beta_{6}^{2}}=\frac{2 \beta_{\mathrm{A}}^{2}}{7 \beta_{\mathrm{A}}^{2} / 6}=\frac{12}{7} .
$$

Let $R_{16} 6(T)$ be the observed ratio of the JND obtained under 2I_1A_X to that obtained under 2I_6A_X at an ISI of $T$ msec. It is commonly assumed that $\Delta F$ is inversely proportional to $d^{\prime}$. Since $d^{\prime}=a /(\sqrt{ } v)$, then $\Delta F=C \sqrt{ } v$, where $C$ is a constant of proportionality. It follows that the ratio $R_{1 \_6}(10)$ at the short 10 -msec ISI should be $\sqrt{ }(12 / 7)$, or 1.31. Table 2, however, shows that the median JND ratio actually observed for the controls is 7.1/4.1, or 1.73. This is larger than Equation 4 predicts.

The assumption of equal components, $\beta_{\mathrm{A}}^{2}=\beta_{\mathrm{X}}^{2}$, is an obvious reason for the failure of Equation 4 . Assume instead that $\beta_{\mathrm{A}}^{2}>\beta_{\mathrm{X}}^{2}$. This inequality of the components of sensory variance might arise from retroactive interference with the processing of the A-stimulus by the immediate arrival of the subsequent X-stimulus. Massaro (1970,1971, 1972) reported a retroactive interference effect of this sort. He named it backward recognition masking. ${ }^{4}$

Let $\beta_{\mathrm{A}}^{2}=k \beta_{\mathrm{X}}^{2}$, where $k>1$, and let $\beta_{\mathrm{X}}^{2}$ be set to unity. Equation 4 becomes

$$
\frac{\beta_{1}^{2}}{\beta_{6}^{2}}=\frac{\beta_{\mathrm{X}}^{2}(k+1)}{\frac{k \beta_{\mathrm{X}}^{2}}{6}+\beta_{\mathrm{X}}^{2}}=\frac{6+6 k}{6+k} .
$$

Since $R_{1 \_6}^{2}(10)=\beta_{1}^{2} / \beta_{6}^{2}$, we can substitute into Equation 5 and solve for $k$ :

$$
k=\frac{6\left[R_{1 \_6}^{2}(10)-1\right]}{6-R_{1 \_6}^{2}(10)} .
$$

For the controls, $R_{1 \_6}^{2}(10)=1.73^{2}=3.00$. This leads to $k=$ 4.00. The control sensory variance for the A-stimulus 
under 2I_1A_X-10 was, therefore, on average about 4 times that for the $\mathrm{X}$-stimulus. This value seems intuitively acceptable.

A similar calculation of $k$ for the dyslexics completely fails. For these subjects, the average $R_{1}^{2}{ }_{6}$ is 13.49 . This drives the denominator of Equation 5 negative, and $k$ also turns negative. The 2I_6A_X procedure at short ISIs, therefore, apparently triggers some psychological process in dyslexics that differs from optimal averaging. One possibility is stabilization of auditory memory. Hari et al. (1999) suggested that dyslexics suffer from a fault in early auditory memory. This fault could interfere with auditory processing of speech, resulting in inadequate development of phonemic representations that are necessary for skilled reading. The memory deficit itself could result from instabilities in the temporal properties of low-level auditory neural discharges or in the subsequent processing of those properties, even at very short ISIs. In either case, the 2I_6A_X procedure might introduce enough stability to overcome the fault in auditory memory.

\section{Signal Detection Analysis: Trace Variance}

Given a value for $k$, we can estimate $A$ in Equation 1 . For the 2I_1A_X paradigm, that equation becomes

$$
v_{1}=\left(k \beta_{\mathrm{X}}^{2}+\beta_{\mathrm{X}}^{2}+A T\right) .
$$

For the 2I_6A_X paradigm, we have

$$
v_{6}=\frac{k \beta_{\mathrm{X}}^{2}}{6+\beta_{\mathrm{X}}^{2}+A T} .
$$

Take the ratio $v_{1} / v_{6}$ of Equation 6 to Equation 7 . By an argument like that which yielded Equation 6, we get

$$
A=\frac{6\left[k+1-R_{1 \_6}^{2}(T)\right]-k R_{1 \_6}^{2}(T)}{6 T\left[R_{1 \_6}^{2}(T)-1\right]} .
$$

At the 400- and the 1,000-msec ISIs, the estimates of $A$ (in units of $\mathrm{msec}^{-1}$ ) are 0.0183 and 0.0122 , respectively, for the controls. The two estimates yield an average value of around 0.015 . A smaller value would probably be obtained were ISI held constant within a session. A similar calculation is not possible for the dyslexics, since no value for $k$ is available.

Under 2I_6A_X-400 and 2I_6A_X-1000, our dyslexic subjects yielded significantly larger frequency JNDs than did the controls. According to the MGB model, trace variance in dyslexics must exceed that in controls, at these ISIs. The similarities and differences in the results across controls and dyslexics under the 2I_1A_X and 2I_6A_X conditions can thereby be tentatively explained.

Under the 2I_1A_X paradigm, dyslexics show no statistically significant effect of ISI. Application of the MGB model suggests that they have abnormally high variance in processing immediately successive stimuli in the 2I_1A_X condition. Trace variance also increases faster for dyslexics than for controls. As ISI lengthens in the 2I_1A_X condition, the processing of immediately successive stim- uli becomes more effective, but trace variance grows. At ISIs of $1,000 \mathrm{msec}$ or less under 2I_1 A_X, these two processes effectively cancel in dyslexics. Therefore, ISI does not affect their $\Delta F$ values. In contrast, JNDs are significantly smaller at the 0 -msec ISI than at the 1,000 -msec ISI for controls. They process immediately successive stimuli efficiently but have larger JNDs at longer ISIs as trace variance grows. For both groups, trace variance dominates performance at the 1,000-msec ISI. Dyslexics perform consistently worse than controls at all ISIs under 2I_1A_X, however, because total variance is always larger in the former than in the latter group.

The controls evidently used averaging under 2I_6A_X to reduce total sensory variance. But the abnormally high sensory variance induced in dyslexics by short ISIs diminishes substantially under this paradigm. Both dyslexics and controls improve at short ISIs. The dyslexics, however, now do as well as the controls. At longer ISIs in the 2I_6A_X condition, both groups perform worse than at shorter ISIs. Trace variance increases in each group and impairs performance. Since trace variance grows faster in dyslexics than in controls, dyslexics perform worse than controls under2I_6A_X-400 and 2I_6A_X-1000.At an ISI of $1,000 \mathrm{msec}$, trace variance completely dominates performance under 2I_6A_X, just as it did under 2I_1A_X. Therefore, frequency JNDs obtained under 2I_1A_X1000 , as compared with 2I_6A_X-1000, do not differ statistically for either group.

Larger trace variance in dyslexics seems to exert a significant effect at 300-400 msec or more after the last Astimulus on a trial. At this time, the A-stimulus representation may start to transfer into a further stage of memory. This stage may be a part of working memory (Baddeley, 1986), which is said to govern digit span. In line with this supposition, significant negative correlations occurred between digit span and JNDs for 2I_1A_X-10 and 2I_1A_X400 in dyslexics and between digit span and the difference between JNDs at 10 and 1,000 msec under 2I_6A_X across all subjects.

Does a single neurophysiological mechanism underlie the increased sensory variance and trace variance in dyslexics? Or do separate mechanisms produce these two psychophysical effects? Faulty stimulus processing at an early, low level in the auditory nervous system might also prevent effective transfer to representations in later stages of memory. The fact that dyslexics have significantly smaller JNDs under 2I_6A_X-400 than under 2I_1A_X400 suggests that stabilizing the initial representations of A-stimuli facilitates the transfer process and retards the growth of trace variance in dyslexics. Distinct neural mechanisms, however, could still be responsible for sensory and trace variance and could function abnormally in dyslexics. Our present data do not eliminate this possibility.

\section{REFERENCES}

Ahissar, M., Protopapas, A., Reid, M., \& Merzenich, M. M. (2000). Auditory processing parallels reading abilities in adults. Proceedings of the National Academy of Sciences, 97, 6832-6837. 
BAdDeley, A. D. (1986). Working memory. Oxford: Oxford University Press.

Baldeweg, T., Richardson, A., Watkins, S., Foale, C., \& Gruzilier,J. (1999). Impaired auditory frequency discrimination in dyslexia detected with mismatch evoked potentials. Annals of Neurology, 45, 495-503.

Bradley, L., \& BRyant, P. E. (1983). Categorizing sounds and learning to read: A causal connection. Nature, 301, 419-420.

Cast ro-Caldas, A., Petersson, A. M., Reis, A., Stone-Elander, S., \& INGVAR, M. (1998). The illiterate brain: Learning to read and write during childhood influences the functional organization of the brain. Brain, 121, 1053-1063.

Cornelissen, P., Richardson, A., Mason, A., Fowler, S., \& Stein, J. (1995). Contrast sensitivity and coherent motion detection measures at photopic sensitivity levels in dyslexic readers and controls. Vision Research, 35, 1483-1494.

D'Agostino, R. B., SR., \& Russell, H. K. (1998). Multivariate Bartlett test. In P. Armitage \& T. Colton (Eds.), Encyclopedia of biostatistics (Vol. 4, pp. 2843-2844). Chichester, U.K.: Wiley.

DeWEIRDT, W. (1988). Speech perception and frequency discrimination in good and poor readers. Applied Psycholinguistics, 9, 163-183.

Dolan, T. G., \& SMALl, A. N., JR. (1984). Frequency effects in backward masking. Journal of the Acoustical Society of America, 75, 932-936.

Dougherty, R. F., Cynader, M. S., Bjornson, B. J., Edgell, D., \& GiaschI, D. E. (1998). Dichotic pitch: A new stimulus distinguishes normal and dyslexic auditory function. NeuroReport, 9, 3001-3005.

Durlach, N. I., \& Braida, L. D. (1969). Intensity perception: I. Preliminary theory of intensity resolution. Journal of the Acoustical Society of America, 46, 372-383.

FARmer, M. E., \& KLein, R. M. (1995). The evidence for a temporal processing deficit linked to dyslexia: A review. Psychonomic Bulletin \& Review, 2, 460-493.

France, S. J., Hansen, P. C., Rosner, B. S., \& Stein, J. F. (1997, August). Low-level auditory encoding in developmental dyslexia. Poster session presented at the 4th World Congress on Dyslexia, Halkidiki, Greece.

Galaburda, A., \& Livingstone, M. (1993). Evidence for a magnocellular defect in developmental dyslexia. In P. Tallal et al. (Eds.), Temporal information processing in the nervous system: Special reference to dyslexia and dysphasia (Annals of the New York Academy of Sciences, Vol. 682, pp. 70-82). New York: New York Academy of Sciences.

GeHr, S. E., \& Sommers, M. S. (1999). Age differences in backward masking. Journal of the Acoustical Society of America, 106, 2793-2799.

Green, D. M., \& Swets, J. A. (1966). Signal detection theory and psychophysics. New York: Wiley.

Hari, R, SäÄSkilahti, A., Helenius, P., \& Uutela, K. (1999). Nonimpaired auditory phase-locking in dyslexic adults. NeuroReport, 10, 2347-2348

Hill, N. I., Bailey, P. J., Griffiths, Y. M., \& Snowling, M. J. (1999). Frequency acuity and binaural masking release in dyslexic listeners. Journal of the Acoustical Society of America, 106, L53-L58.

JASTAK, S., \& WILKINSON, D. (1984). Wide-range achievement test (revised). Wilmington, DE: Jastak Associates.

KaERnbach, C. (1991). Simple adaptive testing with the weighted up-down method. Perception \& Psychophysics, 49, 227-229.

Lovegrove, W. (1993). Weakness in the transient visual system: A causal factor in dyslexia? In P. Tallal et al. (Eds.), Temporal information processing in the nervous system: Special reference to dyslexia and dysphasia (Annals of the New York Academy of Sciences, Vol. 682, pp. 57-69). New York: New York Academy of Sciences.

Macmillan, N. A., Goldberg, R. F., \& Braida, L. D. (1988). Resolution for speech sounds: Basic sensitivity and context memory on vowel and consonant continua. Journal of the Acoustical Society of America, 84, 1262-1280.

MAIR, V. H. (1996). Modern Chinese writing. In P. T. Daniels \& W. Bright (Eds.), The world's writing systems (pp. 200-208). Oxford: Oxford University Press.

Massaro, D. W. (1970). Preperceptual auditory images. Journal of Experimental Psychology, 85, 411-417.
Massaro, D. W. (1971). Effect of masking tone duration on preperceptual auditory images. Journal of Experimental Psychology, 87, 146-148.

Massaro, D. W. (1972). Preperceptual images, processing time, and perceptual units in auditory perception. Psychological Review, 79, 124-145.

Massaro, D. W., \& Burke, D. (1991). Perceptual development and auditory backward recognition masking. Developmental Psychology, 27, 85-96.

McAnally, K. I., \& Stein, J. F. (1996). Auditory temporal coding in dyslexia. Proceedings of the Royal Society of London: Series B, 263, 961-965.

Menell,P., McAnally, K. I., \& Stein, J. F. (1999). Psychophysical sensitivity and physiological response to amplitude modulation in adult dyslexic listeners. Journal of Speech, Language, \& Hearing Research, 42, 797-803.

MoraIs, J. (1985). Literacy and awareness of the units of speech: Implications for research on the units of perception. Linguistics, 23, 705-721.

Nagarajan, S., Mahncke, H., Salz, T., Tallal, P., Roberts, T., \& Merzenich, M. M. (1999). Cortical auditory signal processing in poor readers. Proceedings of the National Academy of Sciences, 96, 6483-6488.

Pennington, B. F., Van Orden, G. C., Smith, S. D., Green, P. A., \& HaIth, M. M. (1990). Phonological processing skills and deficits in adult dyslexics. Child Development, 61, 1753- 1778.

Randles, R. H., \& Wolfe, D. A. (1980). Introduction to the theory of nonparametric statistics. New York: Wiley.

Read, C., Zhang, Y.-F., Nie, H.-Y., \& Ding, B.-Q. (1986). The ability to manipulate speech sounds depends on knowing alphabetic writing. Cognition, 24, 31-44.

Roush, W. (1995). Arguing over why Johnny can't read. Science, 267, 1896-1898.

Rutter, M., \& Yule, W. (1975). The concept of specific reading retardation. Journal of Child Psychology \& Psychiatry, 16, 181-197.

Shankweiler, D., Liberman, I. Y., Mark, L. S., Fowler, C. A., \& FisCHER, F. W. (1979). The speech code and learning to read. Journal of Experimental Psychology: Human Learning \& Memory, 5, 531-545.

Shaywitz, S. E., Shaywitz, B. A., Fletcher, J. M., \& Escobar, M. D. (1990). Prevalence of reading disability in boys and girls: Results of the Connecticut longitudinal study. Journal of the American Medical Association, 264, 998-1002.

Siegel, L. (1992). An evaluation of the discrepancy definition of dyslexia. Journal of Learning Disabilities, 25, 619-629.

Skottun, B. (2000). The magnocellular deficit theory of dyslexia: The evidence from contrast sensitivity. Vision Research, 40, 111-127.

Stanovich, K. (1991). Discrepancy definitions of reading disability: Has intelligence led us astray? Reading Research Quarterly, 26, 7-29.

STEIN, J. F. (1993). Dyslexia: Impaired temporal information processing? In P. Tallal et al. (Eds.), Temporal information processing in the nervous system: Special reference to dyslexia and dysphasia (Annals of the New York Academy of Sciences, Vol. 682, pp. 83-86). New York: New York Academy of Sciences.

Stein, J. F., \& McAnally, K. (1995). Auditory temporal processing in developmental dyslexics. Irish Journal of Psychology, 16, 220-228.

Stein, J. F., Richardson, A. J., \& Fowler, M. S. (2000). Monocular occlusion can improve binocular control and reading in dyslexics. Brain, 123, 164-170.

Stein, J. [F.], \& WALSH, V. (1997). To see but not to read; the magnocellular theory of dyslexia. Trends in Neurosciences, 20, 147-152.

Talcott, J., Hansen, P., Elikim, A., \& Stein, J. (2000). Visual motion sensitivity in dyslexia: Evidence for temporal and energy integration. Neuropsychologia, 38, 935-943.

Talcott, J., Hansen, P., Willis-Owen, C., McKinnell, I., RichardSON, A., \& STEIN, J. (1998). Visual magnocellular impairment in adult developmental dyslexics. Neuro-ophthamology, 20, 187-201.

TAllal, P. (1980). Auditory temporal perception, phonics, and reading disabilities in children. Brain \& Language, 9, 192-198.

TAllal,P., \& Piercy, M. (1973). Developmental aphasia: Impaired rate of non-verbal processing as a function of sensory modality. Neuropsychologia, 11, 389-398.

TAllal, P., \& Piercy, M. (1974). Developmental aphasia: Rate of audi- 
tory processing and selective impairment of consonant perception. Neuropsychologia, 12, 83-93.

Torgeson, J. K. (1978). Performance of reading disabled children on serial memory tasks: A review. Reading Research Quarterly, 19, 57-87.

Turner, M. (1997). Psychological assessment of dyslexia. London: Whurr.

Vellutino, F. R. (1987). Dyslexia. Scientific American, 256, 20-27.

Vellutino, F. R., Steger, J. A., \& Kandel, A. (1972). Reading disability: An investigation of the perceptual deficit hypothesis. Cortex, $\mathbf{8}$, 106-118.

WECHSLER, D. (1981). Wechsler adultintelligence scales (revised). San Antonio, TX: Psychological Corporation and Harcourt Brace Jovanovich.

Weir, C. C., Jesteadt, W., \& Green, D. M. (1977). Frequency discrimination as a function of frequency and sensation level. Journal of the Acoustical Society of America, 61, 178-184.

Werner, L. A., \& GRAY, L. (1998). Behavioral studies of hearing development. In E. W. Rubel, A. N. Popper, \& R. R. Fay, (Eds.), Springer handbookof auditory research (Vol. 9, pp. 12-79). New York: SpringerVerlag.

Witton, C., Talcott, J. B., Hansen, P. C., Richardson, A. J., Griffiths, T. D., Rees, A., Stein, J. F., \& Green, G. G. R. (1998). Sensitivity to dynamic auditory and visual stimuli predicts nonword reading ability in both dyslexic and normal readers. Current Biology, 8, 791-797.

Wright, B. A., Lombardino, L. J., King, W. M., Puranik, C. S., Leonard, C. M., \& Merzenich, M. M. (1997). Deficits in auditory temporal and spectral resolution in language-impaired children. $\mathrm{Na}$ ture, 387, 176-178.

\section{NOTES}

1. These various auditory abnormalities seem too small to influence speech perception. They may indicate more serious disturbances that can hinder language acquisition, analogous to the apparent situation in the visual domain. Perceptual deficits of the size observed in adults could have a large effect on the acquisition of complex skills in childhood, when the central nervous system is immature. Although some auditory functions, such as filter bandwidth, mature in the 1st year of life, others, such as frequency discrimination, do not develop fully until the early school years (see Werner \& Gray, 1998). Massaro and Burke (1991) reported another example of delayed maturation. Children $61 / 2$ years in age needed a larger intensity difference than do adults before they could ac- curately classify the tones as soft or loud; once intensity differences were adjusted, however, the children showed an adult temporal course for backward recognition masking (Massaro, 1970, 1971, 1972).

2. The failure of the transformed 2I_6A_X data to meet Box's test increases the probability of a Type II error in between-group comparisons. Had Box's test been satisfied, order or status $\times$ order might have proved statistically significant. The marginal means for order indicated that subjects who had experienced 2I_1A_X before 2I_6A_X might have done better on the latter, an apparent practice effect. The marginal means for status $\times$ order suggested that the controls might have produced most of this effect. Box's test, however, is sensitive to some types of nonnormality that do not affect GLMs. The test is not recommended as a routine diagnostic (D'Agostino \& Russell, 1998).

3 . For corroboration, we expanded the dyslexic group to include the 3 subjects previously eliminated for high error rates on check trials. We then repeated all the statistical analyses. There were a few minor changes from the prior analyses that used the data from only 16 dyslexics. For 2I_6A_X data, the GLM analysis showed that order $[F(1,36)=3.950$, $p=.055]$ and status $\times$ order $[F(1,36)=3.555, p=.067]$ were closer to significance than previously. Reanalysis of the data combined over 2I_1A_X and 2I_6A_X produced a barely significant interaction of order and condition [Greenhouse-Geiser $F(1,35)=5.875, p=.021]$. Those subjects who experienced 2I_1A_X first showed more improvement under 2I_6A_X than did the subjects who underwent the conditions in the opposite order. (Mauchly's test of sphericity for ISI $\times$ order had become significant, requiring reliance on the Greenhouse-Geiser $F$.) Check trials under 2I_6A_X were now significantly worse for the dyslexics than for the controls $(U=99.5, N=39, p=.01)$. Spearman correlation coefficients increased between JNDs for the dyslexics, both within and between conditions. Thirteen were now significant at the .01 level, and another six were significant at the .025 level. The dyslexics' reading scores now correlated significantly with JNDs for 2I_1A_X-400 and 2I_1A_X-1000, as did digit span.

4. Backward recognition masking should not be confused with backward masking, which is greater in language-impaired than in normal children (Wright et al., 1997). Backward masking occurs in a detection task. It has a much shorter time course than does backward recognition masking, which occurs with suprathreshold stimuli. The bulk of backward masking in young adults occurs at ISIs of $20 \mathrm{msec}$ or less (see, e.g., Dolan \& Small, 1984; Gehr \& Sommers, 1999). In contrast, backward recognition masking remains strong at ISIs of up to $100 \mathrm{msec}$ or so (Massaro, 1972).

APPENDIX A

Spearman Correlation Coefficients for Control Subjects $(n=20)$

\begin{tabular}{|c|c|c|c|c|c|c|c|c|}
\hline & \multicolumn{4}{|c|}{ 2I_1A_X $\Delta F$} & \multicolumn{4}{|c|}{ 2I_6A_X $\Delta F$} \\
\hline & 0 -msec ISI & 10-msec ISI & 400-msec ISI & 1,000 -msec ISI & 10-msec ISI & 200-msec ISI & 400-msec ISI & 1,000 -msec ISI \\
\hline \multicolumn{9}{|l|}{ 2I_1A_X Condition } \\
\hline$\overline{10}-\overline{m s e c}$ ISI & $.85 * *$ & & & & & & & \\
\hline 400-msec ISI & $.68 * *$ & $.60 * *$ & & & & & & \\
\hline $1,000-\mathrm{msec}$ ISI & $.69 * *$ & $.74 * *$ & $.82 * *$ & & & & & \\
\hline \multicolumn{9}{|l|}{ 2I_6A_X Condition } \\
\hline 10-msec ISI & $.66^{* *}$ & $.66^{* *}$ & .46 & $.68 * *$ & & & & \\
\hline 200-msec ISI & $.56^{* *}$ & $.62 * *$ & .47 & $.74 * *$ & $.73 * *$ & & & \\
\hline 400-msec ISI & .46 & .49 & .44 & $.58 * *$ & $.78 * *$ & $.55^{*}$ & & \\
\hline 1,000 -msec ISI & .44 & .50 & $.53^{*}$ & $.61 * *$ & $.73 * *$ & $.71 * *$ & $.70 * *$ & \\
\hline \multicolumn{9}{|c|}{ Psychometric Variable } \\
\hline Reading & -.33 & -.27 & -.50 & -.22 & .13 & .04 & -.34 & -.45 \\
\hline Spelling & -.01 & .02 & -.30 & .02 & .12 & .20 & -.10 & -.14 \\
\hline Digit span & -.36 & -.19 & -.41 & -.15 & -.14 & .23 & -.30 & -.17 \\
\hline Block design & -.31 & -.32 & -.11 & .04 & -.14 & .24 & -.27 & .10 \\
\hline Pictures & -.21 & -.06 & -.34 & -.32 & -.07 & -.26 & -.07 & $-.51 *$ \\
\hline
\end{tabular}

$* p<.025 ; * * p<.01$. 
APPENDIX B

Spearman Correlation Coefficients for Dyslexic Subjects $(n=16)$

\begin{tabular}{|c|c|c|c|c|c|c|c|c|}
\hline & \multicolumn{4}{|c|}{ 2I_1A_X $\Delta F$} & \multicolumn{4}{|c|}{ 2I_6A_X $\Delta F$} \\
\hline & 0 -msec ISI & 10-msec ISI & 400-msec ISI & 1,000 -msec ISI & 10-msec ISI & 200-msec ISI & 400-msec ISI & 1,000-msec IS \\
\hline \multicolumn{9}{|c|}{ 2I_1A_X Condition } \\
\hline $10-\mathrm{msec}$ ISI & $.83^{* *}$ & & & & & & & \\
\hline 400-msec ISI & $.75^{* *}$ & $.84 * *$ & & & & & & \\
\hline $1,000-\mathrm{msec}$ ISI & .50 & .42 & $.65^{* *}$ & & & & & \\
\hline \multicolumn{9}{|l|}{ 2I_6A_X Condition } \\
\hline $10-\mathrm{msec}$ ISI & .54 & .46 & .57 & .46 & & & & \\
\hline 200-msec ISI & .42 & .40 & $.60^{*}$ & .57 & $.60^{*}$ & & & \\
\hline 400-msec ISI & .42 & .20 & .40 & .53 & $.63 * *$ & .50 & & \\
\hline 1,000 -msec ISI & .48 & .34 & .57 & .54 & .58 & $.73^{* *}$ & $.79 * *$ & \\
\hline \multicolumn{9}{|c|}{ Psychometric Variable } \\
\hline Reading & -.41 & -.50 & $-.68 * *$ & -.58 & -.48 & $-.60 *$ & -.49 & $-.59 *$ \\
\hline Spelling & -.07 & -.21 & -.44 & -.48 & -.29 & -.35 & -.37 & -.28 \\
\hline Digit span & -.45 & -.58 & $-.62 * *$ & -.51 & -.55 & -.53 & -.36 & -.46 \\
\hline Block design & -.02 & -.01 & -.09 & -.14 & -.06 & .20 & -.15 & .17 \\
\hline Pictures & .01 & -.09 & -.07 & .01 & .47 & .22 & -.04 & .03 \\
\hline
\end{tabular}

$* p<.025 ; * * p<.01$

(Manuscript received December 31, 2000; accepted for publication April 25, 2001.) 